\title{
The concept of matric flux potential applied to simulation of evaporation from soil
}

\author{
C. F. Shaykewich ${ }^{1}$ and L. Stroosnijder \\ Department of Theoretical Production Ecology, Agricultural University, Wage- \\ ningen, the Netherlands
}

Accepted: 17 February 1977

Key words: Matric flux potential, simulation, evaporation

\section{Summary}

Models for evaporation of water from soil with matric water potential and hydraulic conductivity independently, and with the concept of matric flux potential were compared. It was shown analytically that the model with matric flux potential provides a more exact estimate of water movement in the soil. This leads to a more exact estimate of evaporation.

Mathematical analysis showed that the estimate of soil water movement, and hence evaporation, is higher by the hydraulic conductivity/water potential method than by the matric flux potential method. This analysis also showed that the difference between methods tends to be greatest in coarse-textured soils. Finally, the analysis suggested that the matric flux potential method would provide the same degree of accuracy with a larger compartment thickness and, consequently, require less computer time. All of these conclusions were verified by the results of simulation runs.

\section{Introduction}

The concept of the matric flux potential provides an interesting approach to the problem of estimating water flow in unsaturated soils. This is particularly true of the case of simulation of evaporation where gravity is often not a significant component of the total soil water potential. It was first introduced by Gardner (1958). An example of the application of this concept is the work of Raats (1971). Warrick (1974) refers to other applications.

The matric flux potential $\left(\mathrm{m}^{2} \mathrm{~s}^{-1}\right)$ may be defined by:

$$
\operatorname{MFLP}(\theta) \equiv \int_{\psi_{0}}^{\psi} \mathrm{k}(\alpha) \mathrm{d} \alpha \equiv \int_{\theta_{0}}^{\theta} \mathrm{D}(\beta) \mathrm{d} \beta
$$

1 Permanent address: Department of Soil Science, University of Manitoba, Winnipeg, Canada. 
where $\mathrm{k}(\alpha)$ is the hydraulic conductivity $\left(\mathrm{m} \mathrm{s}^{-1}\right.$ ) as a function of matric water potential $\alpha$ (m of water), and $\mathrm{D}(\beta)$ is the water diffusivity $\left(\mathrm{m}^{2} \mathrm{~s}^{-1}\right)$ as a function of the water content $\beta$. The integration is performed between some upper limit of matric water potential $\psi_{0}$ at which the water content is $\Theta_{0}$ and the desired matric water potential $\psi$ at which the water content is $\Theta .{ }^{2}$ The matric flux potential may be expressed as a function of matric water potential or water content, although it would appear to be most useful when expressed as a function of water content.

The matric flux potential has the unique property that it combines both parameters controlling water flow (hydraulic conductivity and water potential) into one parameter. In estimating water flow in very dry soils, one often must calculate a finite water flux as the mathematical product of a negligible hydraulic conductivity and an infinitely large potential gradient. Calculations involving the product of an infinitely small and an infinitely large number are always open to question. The use of matric flux potential eliminates this problem. To appreciate other advantages of the use of this concept in simulation modeling of evaporation, it is useful to consider some of the history of simulation of water flow in soils.

Soil water flux may be described by:

$$
\mathrm{J}^{\mathrm{V}}=-\mathrm{k}(\psi) \Delta \psi_{\mathrm{t}}
$$

where $\mathbf{J}^{\mathbf{v}}$ is the flux $\left(\mathrm{m} \mathrm{s}^{-1}\right)$ and $\psi_{\mathrm{t}}$ is the total soil water potential including gravity. The other symbols have the same meaning and dimensions as in Eq. 1.

In cases where gravity can be ignored, such as computation of evaporation from dry soil, and the flux is in one direction only, Eq. 2 can be simplified into:

$$
\mathrm{J} \mathrm{V}=-\mathrm{k}(\psi) \frac{\delta \psi}{\delta \mathrm{z}}
$$

where $\mathrm{z}$ is the vertical coordinate taken as positive in the upward direction.

Digital simulation of water flow in soils inherently leads to a division of the soil profile into a number of compartments of some specified thickness. Irrespective of the kind of simulation performed or the simulation language used, the simulation is accomplished by calculating fluxes over finite time steps and using these to estimate changes in soil water content. Thus, the procedure is a kind of sophisticated bookkeeping of a number of compartments.

Due to the nature of the simulation, one has no alternative but to keep the fluxes constant during an individual time step. Thus, one assumes a situation of steady state between two points in the soil at some finite distance $z_{2}-z_{1}=\Delta z$, and approximates Eq. 3 by:

$$
\mathrm{J} \mathrm{v}=-\overline{\mathrm{k}} \frac{\Delta \psi}{\Delta \mathrm{z}}
$$

2 In his work, Raats (1971) chose a reference potential $\psi_{0}$ of $-\infty$. This is not convenient for calculating MFLP because usually neither hydraulic conductivity nor water potential are known at very low water contents. In estimating water movement only the gradient and not the absolute value of matric flux potential is required. Hence for purposes of this study, it was found most convenient to define $\psi_{0}$ as an upper limit of water potential, e.g. $-0.1 \mathrm{~m}$ of water. 
where $\mathrm{J}^{\mathrm{v}}$ is now the flux across the boundary between the two compartments, and $\overline{\mathrm{k}}$ is an 'average' hydraulic conductivity between $z_{1}$ and $z_{2}$ where the water contents are $\Theta_{1}$ and $\Theta_{2}$, respectively. Having already made the assumptions of steady state and an 'average' hydraulic conductivity, there seems little reason to be concerned about having no better way to estimate the potential gradient than by dividing the finite difference $\Delta \psi$ by $\Delta z$.

Apart from the above assumptions, one has to deal with the problem of choosing an appropriate time interval over which the calculation is to be made. Obviously, the smaller the time interval the better the estimate of water movement. However, if the time interval is very small, one soon encounters the practical problem of excessive computer time requirement. This problem can be minimized by using a Continuous System Modeling Program (IBM, 1972) with a variable time step method of integration. Such a program chooses the largest time interval permissible for a given error. This system also has the advantage that the time interval can be changed as the simulation proceeds, thus avoiding small time intervals in stages of the simulation where they are not needed.

It is at this point that the advantage of using matric flux potential can be demonstrated. If one were to write a simulation of steady state water flow using this concept, it would be represented by:

$$
\mathrm{J} v=-\frac{1}{\Delta z} \int_{\psi_{1}}^{\psi_{2}} \mathrm{k}(\psi) \mathrm{d} \psi==-\frac{1}{\Delta \mathrm{z}}(\operatorname{MFLP}(2)-\operatorname{MFLP}(1))
$$

The extreme right-hand side of Eq. 5 is the difference in matric flux potential between the two matric water potentials $\psi_{2}$ and $\psi_{1}$, and is an exact representation of the integral.

Thus the estimate of water flow in a simulation model with the matric flux potential should more closely approach the real answer than a model using matric water potential and hydraulic conductivity independently. It also has the distinct advantage that any uncertainty as to the best procedure for estimating $\bar{k}$, referred to by de Wit \& van Keulen (1972), is removed.

If one wishes to improve the accuracy of a simulation model employing Continuous System Modeling Program (CSMP), one can do so most easily by decreasing the value of $\Delta \mathrm{z}$. There are, however, some practical limits to which $\Delta \mathrm{z}$, the compartment thickness, can be reduced. These are usually determined by the computer time one has at his disposal. A decrease in compartment thickness means that more mathematical operations have to be conducted for the same depth of soil. Also, as demonstrated by de Wit \& van Keulen (1972), the time constant of the simulation is decreased with the result that more computer time is required per unit of simulated time. Therefore, there are instances in which decreasing compartment thickness is not a practical solution to the problem of achieving sufficient accuracy.

The use of the matric flux potential provides an alternative method of achieving better accuracy. It has been shown that the matric flux potential is a more exact method of simulating water movement than using hydraulic conductivity and water 
potential independently. If this is true, one should be able to achieve the same accuracy with a larger compartment thickness (thus using less computer time) with a model using the matric flux potential.

It has been shown above that the model with the matric flux potential provides a more exact estimate of water movement. It is also of interest to determine whether this more exact method gives an estimate which is higher or lower than the conventional method. This can be accomplished through some simple mathematical analysis.

Consider the case where hydraulic conductivity is related to matric water potential by: ${ }^{3}$

$$
\mathrm{k}=\mathrm{a} \mathrm{e}^{x \psi}
$$

With Darcy's Law:

$$
\mathbf{J} \mathbf{v}=-\mathbf{k} \frac{\delta \psi}{\delta \mathbf{z}}
$$

the steady flow over a finite distance $\Delta \mathrm{z}$ is represented in a simulation model by:

$$
\mathbf{J} \mathbf{v}=-\frac{1}{\Delta z} \int_{\psi_{1}}^{\psi_{2}} \mathrm{k} \mathrm{d} \psi
$$

In models with hydraulic conductivity and matric water potential independently, the equation becomes:

$$
\mathbf{J}^{\mathrm{V}}=-\frac{1}{\Delta \mathrm{z}} \mathrm{k} \int_{\psi_{1}}^{\psi_{2}} \mathrm{~d} \psi=-\frac{1}{\Delta z} \mathrm{k}\left(\psi_{2}-\psi_{1}\right)
$$

If one assumes that $\mathrm{k}$ is the simple linear average of the hydraulic conductivities of the two adjacent compartments, then it follows that:

$$
\mathrm{J} V=-\frac{1}{\Delta \mathrm{z}} \frac{\left(\mathrm{ae}^{\alpha \psi_{1}}+\mathrm{ae}^{\alpha \psi 2}\right)}{2}\left(\psi_{2}-\psi_{1}\right)
$$

On the other hand, if matric flux potential is used:

$$
\begin{aligned}
& \mathrm{J} V=-\frac{1}{\Delta \mathrm{z}} \int_{\psi_{1}}^{\psi_{2}} \mathrm{k} \mathrm{d} \psi=-\frac{1}{\Delta \mathrm{z}} \int_{\psi_{1}}^{\psi_{2}} \mathrm{ae}^{\alpha \psi} \mathrm{d} \psi \\
& =\frac{1}{\Delta \mathrm{z}}\left(\frac{\mathrm{ae}^{\alpha \psi_{2}}}{\alpha}-\frac{\mathrm{ae}^{\alpha \psi_{1} 1}}{\alpha}\right)
\end{aligned}
$$

The ratio of water flow by the two methods can now be calculated. A convenient form of the equation of the resulting ratio is:

3 Although this function often describes $\mathrm{k}(\psi)$ over a limited wet range only (Rijtema, 1965), the value of $\alpha$ reflects the general steepness of the $\mathrm{k}-\psi$ relationship and therefore can be used for an approximate analysis. 


$$
\frac{\mathrm{K}-\mathrm{S}}{\mathrm{MFLP}}=\frac{\alpha}{2}\left(\psi_{1}-\psi_{2}\right)\left(1+\frac{2 \mathrm{e}^{\alpha \psi_{2}}}{\mathrm{e}^{\alpha \psi_{1}} \mathrm{e}^{\alpha \psi_{2}}}\right)
$$

where K-S and MFLP refer to the hydraulic conductivity/water potential and matric flux potential methods, respectively.

At this point it is possible to evaluate the ratio if the values of $\alpha$ and water potentials in two adjacent compartments are known. Stroosnijder (1976) reported for 20 soils ranging in texture from coarse sand to clay that the values of $\alpha$ ranged from 0.2 to $0.02 \mathrm{mbar}^{-1}$. If one gives $\alpha$ the value of 0.2 in the above equation, it is immediately clear that in cases where the difference in water potential between two adjacent compartments is 10 mbar or more, the $\mathrm{K}-\mathrm{S}$ method results in greater water flow. If one moves to the other end of the range of possible values for $\alpha$, e.g. 0.02 , then by similar reasoning it is obvious that the $\mathrm{K}-\mathrm{S}$ method will give a higher estimate of water flow in all instances where the difference in water potential is 100 mbar or more.

From the nature of the equation, it would appear that it is at the low values for $\alpha$ that the two methods might tend to be equal. Therefore, it is useful to perform the calculation in which $a=0.02, \psi_{2}=-110 \mathrm{mbar}$, and $\psi_{1}=-100 \mathrm{mbar}$. The resulting ratio is 1.0033 , i.e. the $\mathrm{K}-\mathrm{S}$ method gives only slightly higher water flow under these conditions. Thus, it is clear that most conditions likely to occur in the soil during the process of evaporation (where gradients in water potential near the surface are likely to be large) will result in a larger estimate of water flow by the K-S method than by the MFLP method.

On the basis of the above analysis, it is possible to make some predictions about the behavior of models simulating evaporation using the two methods of estimating water flow.

1. During evaporation, there will be less water movement and thus lower evaporation in the model using the MFLP method.

2. The MFLP method will provide the same degree of accuracy in estimating soil water movement with a larger compartment thickness and consequently require less computer time for results of the same degree of accuracy.

3. Mathematical analysis revealed that the lower the value of $\alpha$, the smaller is the difference between the K-S and MFLP methods. Since small values of $\alpha$ are usually associated with fine textured soils, the differences between methods will be least pronounced in fine textured soils. Coarse textured soils, which usually have large values of $\alpha$, will show the greatest differences.

\section{Procedure}

\section{Description of the simulation program}

The simulation program was similar to that presented by van Keulen (1975), i.e. the soil was divided into a number of compartments. The procedure was to simulate evaporation of water from the soil surface and the consequent movement of water within the soil profile. The total depth of soil used was $50 \mathrm{~cm}$. This was considered 
adequate since there is some evidence that the depth of zero flux during evaporation will seldom be greater than $30 \mathrm{~cm}$ (Jackson et al., 1973).

Three combinations of compartment thickness were compared. Starting from the soil surface, the first combination consisted of 5 compartments of $1 \mathrm{~cm}, 5$ of $1.5 \mathrm{~cm}, 3$ of $2.5 \mathrm{~cm}, 4$ of $5 \mathrm{~cm}$, and 1 of $10 \mathrm{~cm}$. The second combination was composed of compartments twice as thick as those of the first, with the last 4 compartments being removed in order to maintain the total depth of $50 \mathrm{~cm}$. The third combination, in turn, was composed of compartments twice the thickness of those in the second combination. Again, the appropriate number of lower compartments were removed in order to maintain a total depth of $50 \mathrm{~cm}$.

The procedure for the evaporation portion of the model was based on an approach presented by Staple (1971). He suggested that the evaporative flux, q, could be related to the difference between vapor pressure at the soil surface, $e_{s}$, and that in the air, $e_{i}$, and a factor $F$ which would account for the effects of wind speed. One might interpret $F$ to be related to the reciprocal of the thickness of the laminar layer of air above the soil. In equation form, the relationship is:

$$
\mathrm{q}=\mathrm{F}\left(\mathrm{e}_{\mathrm{s}}-\mathrm{e}_{\mathrm{a}}\right)
$$

For purposes of this study, $\mathrm{e}_{\mathrm{a}}$ was given a value of 7.06 mbar. This corresponds to an atmosphere at $20{ }^{\circ} \mathrm{C}$ and $30 \%$ relative humidity. The temperature at the soil surface was assumed to be $25^{\circ} \mathrm{C}$. At this temperature, $\mathrm{e}_{\mathrm{s}}$ was calculated from water potential at the soil surface using the classical relationship between water potential and relative vapour pressure. The saturation vapour pressure at $25^{\circ} \mathrm{C}$ is 31.45 mbar. Using this value one can calculate the value of $\mathrm{F}$ in $\mathrm{Eq} .14$ required to give any predetermined 'potential evaporation rate'. The potential evaporation rate used in this study was $0.8 \mathrm{~cm} /$ day, with the result that $F$ was given a value of 0.0328 .

Evaporative demand was assumed to be constant over time, i.e. there was no adjustment for any diurnal variation in potential evaporation rate such as one might expect in nature. The duration of the simulated evaporation was 5 days and evaporation rate, total accumuated evaporation and water content in each compartment were recorded every 0.25 days.

\section{Calculation of soil water movement}

The calculation of soil water movement was conducted by two methods. The first involved the use of matric water potential/water content and hydraulic conductivity/water content relationships and is hereafter referred to as the K-S method. The second method utilized the relationship between matric flux potential and water content and was given the designation MFLP.

In the K-S method, the difference in matric water potential (mbar) between two compartments was calculated and multiplied by the reciprocal of the distance between centres of the two compartments to give the matric potential gradient (mbar per $\mathrm{cm}$ depth). From this was subtracted the gravitational potential gradient (1 mbar per $\mathrm{cm}$ depth) to give the total potential gradient. Hydraulic conductivity $(\mathrm{cm} /$ day) was calculated from the average water content in each compartment. 
The arithmetic mean of the hydraulic conductivities for the two adjacent compartments was calculated. This was multiplied by the gradient in total potential to calculate flux. The net flux ( $=$ incoming flux - outgoing flux) divided by the thickness of the appropriate compartment yielded the change in water content. This quantity was added to the initial water content to give a new water content which was then used in calculating water flow in the next time step.

The first phase of the MFLP method was the calculation of the matric flux potential/water content relationship for each soil. Matric flux potential was calculated from:

$$
\text { MFLP }=\int_{\psi_{0}}^{\psi} \mathrm{k} \mathrm{d} \psi={ }_{\psi_{0}}^{\psi} \mathrm{k} \Delta \psi
$$

The calculation started by defining the value of $\psi_{0}$, the choice being made in such a way as to insure that the entire range in water content used in the simulation was included. (The reference point of the calculation influences the absolute values

Table 1. Data illustrating the calculation of matric flux potential for Adelanto loam.

\begin{tabular}{|c|c|c|c|c|c|}
\hline $\mathrm{k}\left(\mathrm{cm} \mathrm{day}^{-1}\right)$ & $\Psi$ (mbar) & $\Delta \Psi$ & $\Delta \Psi \times \mathrm{k}_{1}$ & MFLP & $\theta\left(\mathrm{cm}^{3} \mathrm{~cm}^{-3}\right.$ \\
\hline 0.53 & -100 & -10 & -5.3 & -5.3 & 0.3200 \\
\hline 0.42 & -110 & $\begin{array}{l}-10 \\
-10\end{array}$ & -4.2 & -9.5 & 0.3165 \\
\hline 0.36 & -120 & $\begin{array}{l}-10 \\
-10\end{array}$ & -3.6 & -13.1 & 0.3130 \\
\hline 0.31 & -130 & $\begin{array}{l}-10 \\
-10\end{array}$ & -3.1 & -16.2 & 0.3090 \\
\hline 0.28 & -140 & $\begin{array}{l}-10 \\
-10\end{array}$ & -2.8 & -19.0 & 0.3070 \\
\hline 0.24 & -150 & $\begin{array}{l}-10 \\
-10\end{array}$ & -2.4 & -21.4 & 0.3030 \\
\hline 0.18 & -160 & $\begin{array}{l}-10 \\
-10\end{array}$ & -1.8 & -23.2 & 0.3010 \\
\hline 0.17 & -170 & $\begin{array}{l}-10 \\
-10\end{array}$ & --1.7 & -24.9 & 0.2990 \\
\hline 0.14 & -180 & $\begin{array}{l}-10 \\
-20\end{array}$ & -2.8 & -27.7 & 0.2907 \\
\hline 0.11 & -200 & $\begin{array}{l}-20 \\
-20\end{array}$ & -2.2 & -29.9 & 0.2920 \\
\hline 0.075 & -220 & $\begin{array}{l}-20 \\
-20\end{array}$ & -1.5 & -31.4 & 0.2875 \\
\hline 0.062 & -240 & $\begin{array}{r}-20 \\
-20\end{array}$ & -1.24 & -32.64 & 0.2840 \\
\hline 0.052 & -260 & $\begin{array}{r}-20 \\
-20\end{array}$ & -1.04 & -33.68 & 0.2810 \\
\hline 0.045 & -280 & $\begin{array}{l}-20 \\
-20\end{array}$ & -0.9 & -34.58 & 0.2770 \\
\hline 0.034 & -300 & $\begin{array}{r}-20 \\
-40\end{array}$ & -1.36 & -35.94 & 0.2740 \\
\hline 0.024 & -340 & $\begin{array}{l}-40 \\
-60\end{array}$ & -1.44 & -37.38 & 0.2680 \\
\hline 0.015 & -400 & $\begin{array}{l}-00 \\
-100\end{array}$ & -1.5 & -38.88 & 0.2610 \\
\hline 0.008 & -500 & $\begin{array}{l}-100 \\
-100\end{array}$ & -0.8 & -39.68 & 0.2525 \\
\hline 0.0042 & -600 & $\begin{array}{l}-100 \\
-100\end{array}$ & -0.42 & -40.10 & 0.2450 \\
\hline 0.003 & -700 & $\begin{array}{l}-100 \\
-300\end{array}$ & -0.9 & -41.00 & 0.2390 \\
\hline 0.0013 & -1000 & $\begin{array}{r}-500 \\
-500\end{array}$ & -0.65 & -41.65 & 0.2275 \\
\hline 0.00046 & -1500 & $\begin{array}{l}-500 \\
-500\end{array}$ & -0.23 & -41.88 & 0.2160 \\
\hline 0.00026 & -2000 & $\begin{array}{l}-300 \\
-1000\end{array}$ & -0.26 & -42.14 & 0.2075 \\
\hline 0.0001 & -3000 & $\begin{array}{r}1000 \\
-1000\end{array}$ & -0.1 & -42.24 & 0.1970 \\
\hline 0.000054 & -4000 & -2000 & -0.108 & -42.348 & 0.1890 \\
\hline 0.00003 & -6.000 & -4000 & -0.12 & -42.468 & 0.1785 \\
\hline 0.000013 & -10000 & -10000 & -0.13 & -42.598 & 0.1650 \\
\hline 0.0000035 & -20000 & -20000 & -0.07 & --42.668 & 0.1440 \\
\hline
\end{tabular}


of MFLP but since only differences in MFLP are used in the calculation of water movement (Eq. 5), the only restriction on the reference point is that it be high enough to include the necessary range in water content.) The value of hydraulic conductivity at $\psi_{0}$ was determined. In the first step of the example (Table 1), $\psi_{0}$ was set at -100 mbar, and $\Delta \psi$ was given the value of -10 mbar. Then $\mathrm{k} \Delta \psi=0.53$ $\times-10=-5.3$.

Thus the value of the matric flux potential at -100 mbar was -5.3 . The value of $\mathrm{k}$ at -110 mbar was determined. With another water potential increment of -10 mbar, $\mathrm{k} A \psi=0.42 \times-10=-4.2$. This figure was added to the contribution of the first calculation giving a matric flux potential at the water content at -110 mbar of $-4.2-5.3=-9.5$. The water potential was again decreased by 10 mbar and the next contribution to matric flux potential calculated. Following this procedure one finds that each contribution is smaller than the previous one. This is due to the rapid decrease in hydraulic conductivity with decreasing water potential. This means that at lower water potentials, the magnitude of the water potential increments may be substantially enlarged without materially affecting the precision of the calculation of matric flux potential. Indeed, as can be seen from Table 1, the water potential increments may be enlarged to 10 bar and more.

A factor which is not accounted for by the matric flux potential is gravity. In the K-S method, the effect of gravity was taken into account by multiplying the arithmetic mean hydraulic conductivity by -1.0 , the gravitational potential gradient, and adding this 'gravitational' flux to flux due to matric potential gradient. In order to make an exact comparison of the two methods, therefore, the calculation of water flux by the MFLP method involved two components: (1) multiplication of the difference in matric flux potential between two adjacent compartments by the reciprocal of the distance between their centres, and (2) multiplication of the arithmetic mean hydraulic conductivity by -1.0 . All subsequent calculations, such as for soil water content, were the same as those for the K-S method.

The simulation of evaporation and consequent soil water movement was carried out with CSMP developed by IBM (1972). Its use has been illustrated in a number of publications (e.g. de Wit \& van Keulen, 1972). The model used the Fourth Order Runge-Kutta method of integration with a variable time step. In this method the time step is adjusted to the largest value that can be used while limiting the error to $\leqslant 0.01 \%$. Values for hydraulic conductivity, water potential, and matric flux potential were entered as tabulated functions of water content. A listing of the computer program may be found in the Appendix.

\section{Soils}

Five soils were used in this study. The data for hydraulic conductivity and matric water potential as a function of water content were obtained from various sources in the literature. A silty clay and a medium coarse sand (Stroosnijder, 1976) provided the extremes in soil texture. Other soils used were Vernal sandy loam (Nimah \& Hanks, 1973), Adelanto loam (Jackson, 1973), and Wood Mountain clay loam (Staple, 1972). 


\section{Initial conditions}

The choice of initial conditions is always difficult in this kind of study. In this instance, it was decided that the initial conditions should be related to the waterconducting properties of the soil. Thus the initial water content of the entire soil profile was assumed to be that at which the hydraulic conductivity had a value of $0.1 \mathrm{~cm} \mathrm{day}^{-1}$. The values of volumetric water content were $0.4725,0.30,0.2925$, 0.2875 , and 0.1925 for the silty clay, Wood Mountain clay loam, Adelanto loam, Vernal sandy loam, and medium coarse sand, respectively. The corresponding values of matric water potential were $-58,-200,-200,-105$ and -55 mbar, respectively.

\section{Results and discussion}

\section{Matric flux potential}

Data for matric flux potential as a function of water content are shown in Fig. 1. These relationships have several interesting features. For all the soils there is a definite tendency for the matric flux potential to reach some well defined maximum value. The numerical value of this maximum has no significance since it obviously depends upon the value given $\psi_{0}$ in the calculations. (It would be important only where one was concerned with water flow at high water contents, e.g. infiltration, since in that instance $\psi_{0}$ would obviously have to be given the value of 0 ). At high water contents, there is a rapid decrease in matric flux potential with decreasing

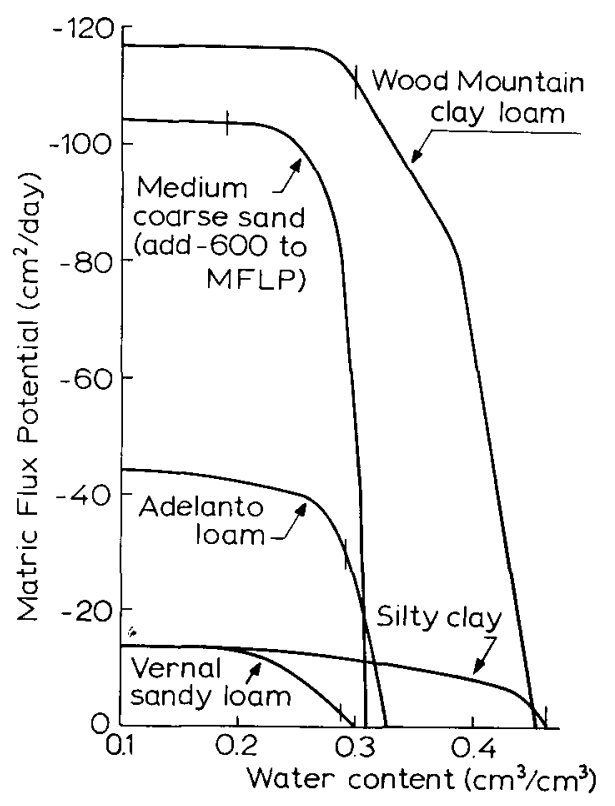

Fig. 1. Relationship of matric flux potential to soil water content for the soils used. For the medium coarse sand, calculation was begun at $\mathbf{- 1 0}$ mbar, for the silty clay at -58 mbar, and at -100 mbar for the other soils. Vertical bars indicate water content at hydraulic conductivity $=0.1 \mathrm{~cm}$ day -1 . 
water content. This region of rapid decrease occurs at different water contents in different soils because of differences in matric water potential and hydraulic conductivity at a given water content. If the water content at the reference potential is sufficiently high, the curve tends to be asymptotic to that water content, e.g. medium coarse sand, Adelanto loam and Wood Mountain clay loam. Thus, the matric flux potential/water content relationships tend to asymptotic to two axes.

In the current simulation, matric flux potential was entered into the simulation model as a tabulated function of water content. In many instances, it may be desireable to enter such data in the form of an aquation rather than as a table of data points. The matric flux potential/water content relationship is a smooth curve and as such it should be possible to express it very accurately in the form of an equation. This is not usually true of the matric water potential/water content and hydraulic conductivity/water content curves. Thus this unique property of the matric flux potential/water content relationship represents another advantage in its use in simulation modeling.

Because the curves are clearly asymptotic to some maximum value, the flux of water in the very low range of water content will be very small, regardless of the magnitude of the gradient in water content. Hence, accurate measurement of matric water potential and hydraulic conductivity in the low water content range is not necessary. Very little error in predicted flux results if one simply extrapolates the curves. Thus, the efforts of many soil scientists to obtain accurate measurements

Table 2. Total evaporation $(\mathrm{cm})$ after 2 and 5 days of simulated evaporation.

\begin{tabular}{|c|c|c|c|c|c|}
\hline \multirow[t]{2}{*}{ Soil } & \multirow{2}{*}{$\begin{array}{l}\text { Top } \\
\text { compartment } \\
\text { thickness }(\mathrm{cm})\end{array}$} & \multicolumn{2}{|l|}{$\mathrm{K}-\mathrm{S} *$} & \multicolumn{2}{|l|}{ MFLP* } \\
\hline & & 2 days & 5 days & 2 days & 5 days \\
\hline \multirow[t]{3}{*}{ Silty clay } & 1 & 1.60 & 3.75 & 1.60 & 3.83 \\
\hline & 2 & 1.60 & 3.87 & 1.60 & 3.88 \\
\hline & 4 & 1.60 & 3.95 & 1.60 & 3.94 \\
\hline \multirow[t]{3}{*}{ Clay Ioam } & 1 & 1.30 & 1.94 & 1.17 & 1.85 \\
\hline & 2 & 1.51 & 2.37 & 1.30 & 2.00 \\
\hline & 4 & 1.59 & 3.11 & 1.56 & 2.29 \\
\hline \multirow[t]{3}{*}{ Loam } & 1 & 1.56 & 2.58 & 1.47 & 2.40 \\
\hline & 2 & 1.59 & 2.92 & 1.57 & 2.60 \\
\hline & 4 & 1.60 & 3.53 & 1.59 & 2.90 \\
\hline \multirow[t]{3}{*}{ Sandy loam } & 1 & 1.60 & 3.41 & 1.60 & 2.81 \\
\hline & 2 & 1.60 & 3.68 & 1.60 & 2.96 \\
\hline & 4 & 1.60 & 3.92 & 1.60 & 3.27 \\
\hline \multirow{3}{*}{$\begin{array}{l}\text { Medium coarse } \\
\text { sand }\end{array}$} & 1 & 0.91 & 1.38 & 0.54 & 0.81 \\
\hline & 2 & 1.18 & 1.67 & 0.63 & 0.89 \\
\hline & 4 & 1.54 & 2.25 & 0.90 & 1.11 \\
\hline
\end{tabular}

* $\mathrm{K}-\mathrm{S}=$ matric water potential/hydraulic conductivity; MFLP = matric flux potential (see text). 
of hydraulic conductivity at very low water contents, although of academic interest, are not likely to contribute significantly to the solution of practical water flow problems.

\section{Evaporation}

Simulated evaporation as affected by simulation method and so:l compartment thickness were studied. Total evaporation is probably the most important parameter to be considered.

Effect of the method of simulating soil water movement. The method of simulating water movement in the soil had a very important influence on the amount of total evaporation (Table 2). With the exception of the silty clay, for a given compartment thickness and soil, the 5-day total evaporation was considerably lower by the MFLP method. Differences in the 2-day accumulated evaporation were usually small or non-existent, mainly because many of the soils were still in the first stage of drying, i.e. actual evaporation $=$ potential evaporation (Table 3). A noteable exception was the medium coarse sand. In this soil, evaporation after 2 days for a $1 \mathrm{~cm}$ top compartment thickness by the MFLP method was only $60 \%$ that by the K-S method. Difference due to method for the other soils were usually not so

Table 3. Duration of the first stage of drying (days).

\begin{tabular}{llll}
\hline Soil & $\begin{array}{l}\text { Top } \\
\text { compartment } \\
\text { thickness }(\mathrm{cm})\end{array}$ & $\mathrm{K}-\mathrm{S}^{*}$ & MFLP* \\
& & \\
Silty clay & 1 & $2.50^{* *}$ & 3.00 \\
& 2 & 2.75 & 3.25 \\
& 4 & 3.75 & 3.75 \\
Clay loam & 1 & 0.75 & 0.75 \\
& 2 & 1.00 & 1.00 \\
& 4 & 1.75 & 1.50 \\
Loam & & & \\
& 1 & 1.50 & 1.25 \\
& 2 & 1.75 & 1.50 \\
Sandy loam & 4 & 2.50 & 2.25 \\
& 1 & & \\
& 2 & 2.50 & 1.75 \\
Medium coarse sand & 1 & 3.25 & 2.25 \\
& 4 & 3.75 & 2.75 \\
& 2 & 0.50 & 0.25 \\
& 4 & 0.75 & 0.50 \\
& & 1.50 & 0.75 \\
\hline
\end{tabular}

* See footnote to table 2 .

** All values are to the nearest quarter of a day. 
Table 4. Computer use time required to stimulate 5 days of evaporation.

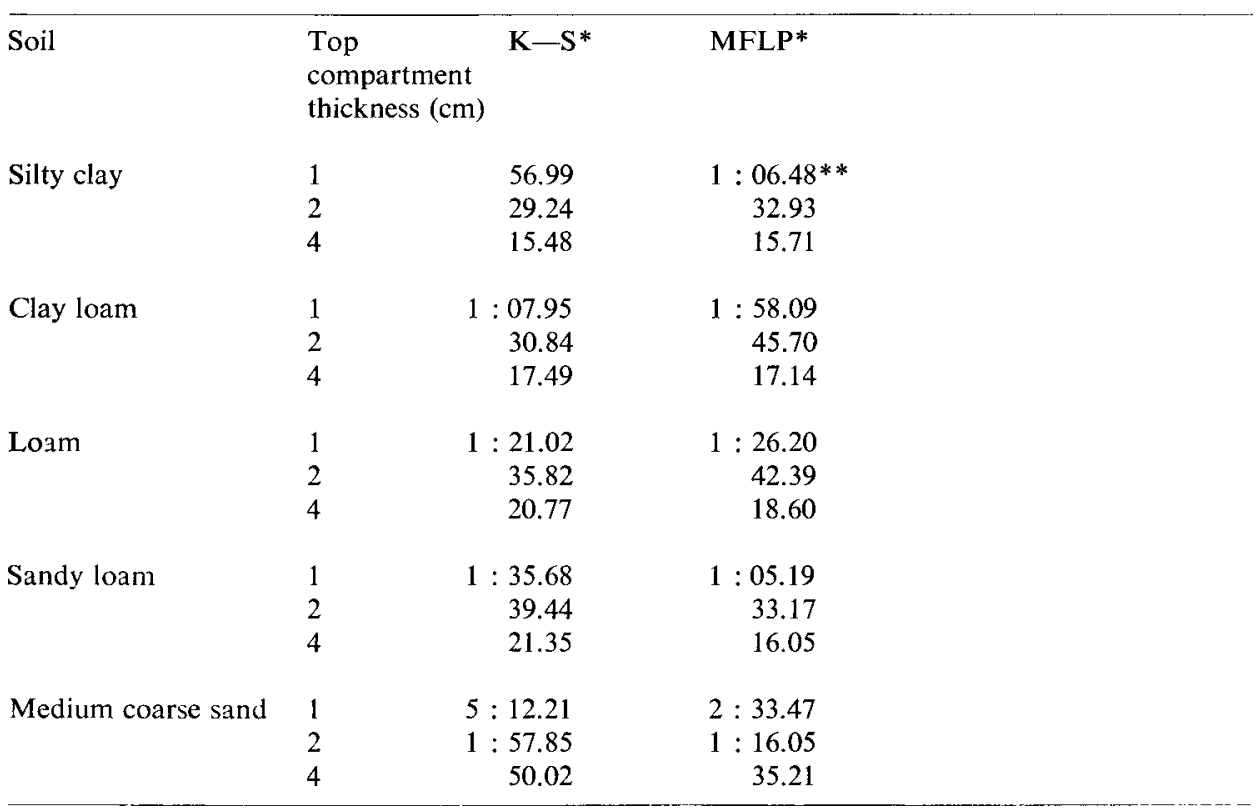

* See footnote to table 2.

** Computer use time is expressed as minutes and seconds, e.g. $1: 06.48=1$ minute, 6.48 seconds.

dramatic, but particularly for the 5-day total evaporation were usually 'significant'.

The lack of difference in the silty clay was in fact predicted by mathematical analyses. It was found that the lower the value of $\alpha$ in the expression relating hydraulic conductivity to matric water potential, the smaller was the difference in calculated soil water movement between the MFLP and K-S methods. It follows that differences in evaporation should also be smaller. Therefore, the relatively small value of $\alpha$ for the silty clay, 0.048 , at least partially accounts for the lack of difference between methods in simulated evaporation in this soil.

The fact that differences between methods occurred in most soils has rather important implications for simulation modeling of evaporation. In the case of the clay loam and loam soils it was possible to obtain the same estimate of evaporation using a $2 \mathrm{~cm}$ top compartment thickness with the MFLP method as with a $1 \mathrm{~cm}$ top compartment thickness with the K-S method. The importance of this result lies in the fact that the MFLP method with a $2 \mathrm{~cm}$ top compartment thickness required about $3 / 4$ of the computer time used by the K-S method with a $1 \mathrm{~cm}$ top compartment thickness (Table 4). Thus if one has a limited computer time at his disposal, one can perform a more accurate simulation by the MFLP method.

For the sandy loam and medium coarse sand, differences between the methods were very large. With the medium coarse sand, for example, even the $1 \mathrm{~cm}$ top compartment thickness with the K-S method gave a higher estimate of evaporation 
than the $4 \mathrm{~cm}$ top compartment thickness with the MFLP method. Also, there was a 10-fold difference in computer time in favour of the MFLP method. Hence, it appears that in coarse-textured soils it is particularly advantageous to use the MFLP method.

Effects of compartment thickness. For each soil within each method, total evaporation is seen to follow a well defined trend with respect to compartment thickness. In almost all cases, total evaporation decreased as the thickness of the top compartment decreased. The exceptions occur at two days in soils which were still in the first stage of drying. Clearly, one does not expect differences in these instances.

The effect of compartment thickness is small in the fine-textured silty clay and tends to become larger as particle size increases. The importance of this trend lies in the acceptability of the error in total evaporation. For example, increasing compartment thickness in the silty clay from 1 to $4 \mathrm{~cm}$ increased total evaporation after 5 days by only $2.5 \%$. It would seem that an error in estimating evaporation of this magnitude would be quite acceptable. On the other hand, increasing compartment thickness from 1 to $4 \mathrm{~cm}$ in the medium coarse sand causes an error of $35 \%$ in the MFLP method and about $50 \%$ in the $\mathrm{K}-\mathrm{S}$ method in total evaporation over 5 days. It is doubtful that one would tolerate errors of this magnitude. Hence, it may be possible to use somewhat larger compartments in fine-textured soils than in coarse-textured soils.

\section{Soil water content profiles}

The differences in total evaporation discussed above are caused eventually by differences in the distribution of water in the soil profile. Also, an examination of soil

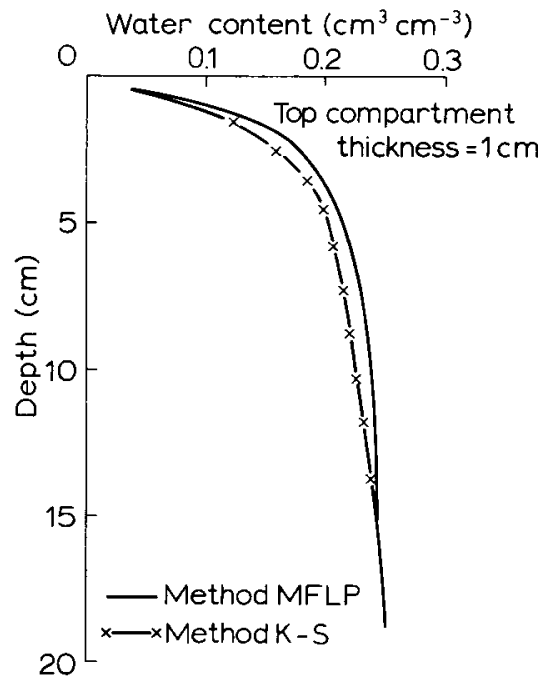

Fig. 2. Simulated water content profiles for Adelanto loam after 5 days of evaporation at a potential evaporation rate of $0.8 \mathrm{~cm}$ day-1. Top compartment thickness $=1 \mathrm{~cm}$. 


\section{F. SHAYKEWICH AND L. STROOSNIJDER}

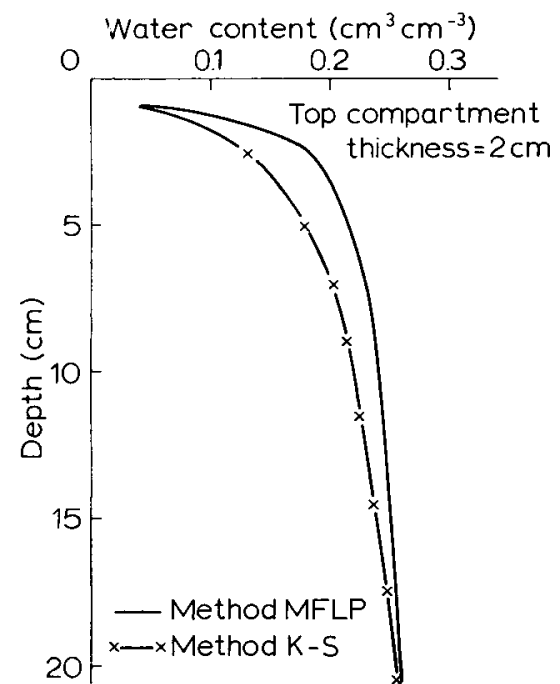

Fig. 3. Simulated water content profiles for Adelanto loam after 5 days of evaporation at a potential evaporation rate of $0.8 \mathrm{~cm}$ day-1. Top compartment thickness $=2 \mathrm{~cm}$.

water content profiles probably gives a better indication of the effects of method and compartment thickness. This conclusion is derived from the work of van Keulen (1975) who found that he could simulate evaporation reasonably accurately but was somewhat less successful in simulating soil water content profiles. For these reasons, soil water content profiles deserve careful consideration in a study of this kind.

The effects of both method and compartment thickness were dependent upon soil texture. The average effect occurred in the average texture, viz. Adelanto loam. Hence, the results of this soil will be discussed in detail, and the effects of soil texture indicated as deviations from the results in this soil.

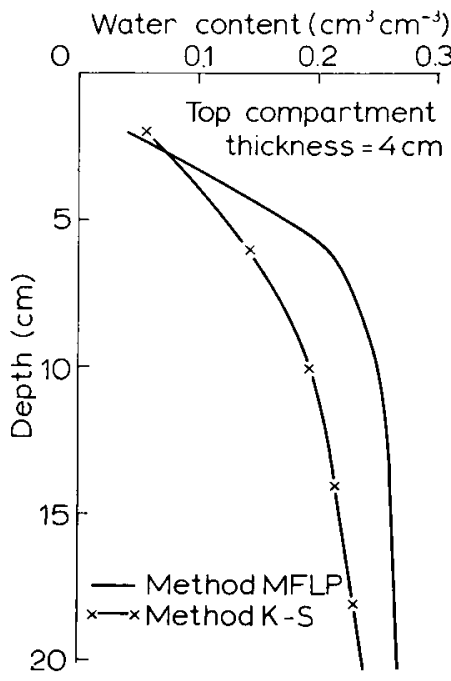

Fig. 4. Simulated water content profiles for Adelanto loam after 5 days of evaporation at a potential evaporation rate of $0.8 \mathrm{~cm}$ day-1. Top compartiment thickeness $=4 \mathrm{~cm}$. 
Effect of the method of simulating soil water movement. The results from the comparison of methods of simulating soil water movement showed that for a given compartment thickness, water content at depths below the soil surface was alway; higher by the MFLP method (Fig. 2-4). It is also noted that with the MFLP method, the gradient in water content near the soil surface was considerably steeper than with the K-S method. The differences between methods were smallest for the $1 \mathrm{~cm}$ top compartment thickness and largest for the $4 \mathrm{~cm}$ top compartment thickness. Thus less error results from using a large compartment thickness with the MFLP method than with the K-S method.

An interesting comparison is that of the water content profile for the 2-cm MFLP method with that for the $1-\mathrm{cm} \mathrm{K-S} \mathrm{method.} \mathrm{In} \mathrm{this} \mathrm{comparison} \mathrm{one} \mathrm{sees} \mathrm{that} \mathrm{the}$ water content profiles are almost identical. It will be recalled that the total evaporation for these two cases was also virtually identical (Table 2). Thus it is shown that in terms of water content profiles, as well as in total evaporation, a $2 \mathrm{~cm}$ top compartment thickness with the MFLP method provides results as accurate as those for the $1 \mathrm{~cm}$ top compartment thickness with the K-S method.

There was an important effect of soil texture on the comparison of the two methods of calculating water movement. In the silty clay after 5 days of simulated evaporation there was no difference in water content profiles between methods for any of the compartment thicknesses. An explanation of this lack of difference in this soil was given earlier. At the coarse end of the texture scale, the Vernal sandy loam and medium coarse sand showed pronounced differences in water content profiles even with the $1 \mathrm{~cm}$ top compartment thickness. This result again illustrated the advantage of using the MFLP method in coarse-textured soils.

Effects of compartment thickness. The effects of compartment thickness were most pronounced in the case of the K-S method (Fig. 5). A smaller compartment thick-

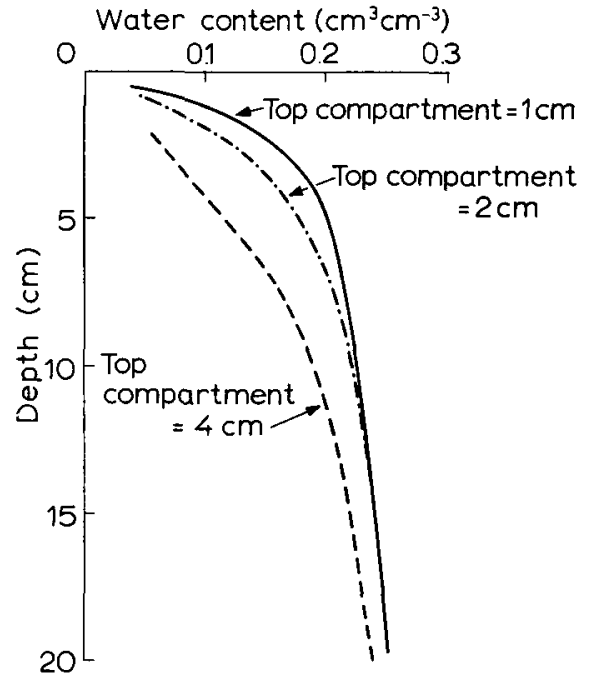

Fig. 5. Simulated water content profiles by the K-S method for Adelanto loam after 5 days of evaporation at a potential evaporation rate of $0.8 \mathrm{~cm}^{\text {day }}-1$. 


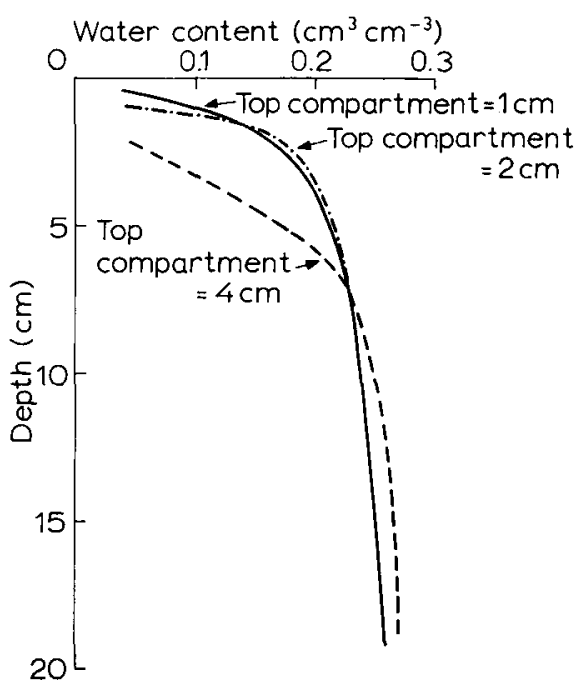

Fig. 6. Simulated water content profiles by the MFLP method for Adelanto loam after 5 days of evaporation at a potential evaporation rate of $0.8 \mathrm{~cm}$ day -1 .

ness resulted in a higher water content at every subsurface depth in the profile. This obviously resulted from the fact that a decrease in compartment thickness caused a decrease in total evaporation. In addition, the larger compartment thickness produced a water content profile in which the rate of change of water content with depth was more uniform than that produced by the smaller compartment thickness. With the smallest compartment thickness, the water content profile is seen to have a very sharp slope in the region of the soil surface. Thus extrapolation of the water content profiles leads to the result that surface water content decreased as compartment thickness decreased. This situation ultimately caused evaporation to decrease with decreasing compartment thickness.

Results obtained with the MFLP method (Fig. 6) showed almost no difference in water content profiles between the $1 \mathrm{~cm}$ and $2 \mathrm{~cm}$ top compartment thicknesses. This indicates that due to the more exact nature of the calculation of water movement by the MFLP method, a compartment thickness of $2 \mathrm{~cm}$ is sufficiently small to provide an adequate estimate of the water content profile in this soil. With the $\mathrm{K}-\mathrm{S}$ method, a top compartment thickness of $1 \mathrm{~cm}$ is required in order to produce a satisfactory water content profile. For the MFLP method, a top compartment thickness of $4 \mathrm{~cm}$ has a much lower water content at a given depth than the other two compartment thickness. Clearly, it does not provide an adequate estimate of the water content profile.

The influence of texture on the effect of compartment thickness showed similar trends for both methods. The Wood Mountain clay loam and the silty clay showed almost no effect of compartment thickness on water content profile. At the other end of the texture range, the medium coarse sand showed a discernible difference between the 1 and $2 \mathrm{~cm}$ top compartment thicknesses, even with the MFLP method. In general, however, the differences between compartment thickness were smaller by the MFLP method than by the K-S method. 


\section{Conclusions}

The utility of the concept of matric flux potential in simulation modelling of evaporation was demonstrated by showing that it provides a more exact estimate of water movement than methods used to date. In the traditional approach, one of the greatest difficulties is deciding upon a method for averaging hydraulic conductivities of adjacent compartments. The use of the matric flux potential eliminated this problem. Another asset of the matric flux potential was that it gives a smooth curve when plotted as a function of water content. This property should make it relatively easy to express matric flux potential as a function of water content very accurately in the form of an equation. Such a step would eliminate the necessity of a function table and thus result in a saving in computer time.

The results of the simulation runs showed that a lower (more accurate) estimate of evaporation was obtained with the MFLP method than with the conventional $\mathrm{K}-\mathrm{S}$ method using an arithmetic mean hydraulic conductivity. Usually a simulation for a $2 \mathrm{~cm}$ top compartment thickness with the MFLP method provided as accurate an estimate of evaporation as one for a $1 \mathrm{~cm}$ top compartment thickness with the $\mathrm{K}-\mathrm{S}$ method. Comparison of computer time required for these two simulations showed that the simulation with the MFLP method usually required about $3 / 4$ the computer time of the $\mathrm{K}-\mathrm{S}$ method. Thus where computer time is a limiting factor, the use of the matric flux potential can provide a better estimate of evaporation.

Differences in evaporation estimates between the two methods were particularly large in coarse-textured soils. Hence, it is suggested that it is especially advantageous to use the MFLP method in coarse-textured soils.

\section{References}

Gardner, W. R., 1958. Some steady state solutions of the unsaturated moisture flow equation with application to evaporation from a water table. Soil Sci. 85; 228-233.

IBM, 1972. Continuous system modeling program III (CSMP III), Program reference manual. Program Product Centre, Dons Mills, Ontario, Canada.

Jackson, R. D., 1973. Diurnal changes in soil water content during drying. In: Field soil water regime. Spec. Publ. Soil Sci. Soc. Am. No 5: 37-55.

Jackson, R. D., B. A. Kimball, R. J. Reginato \& F. S. Nakayama, 1973. Diurnal soil water evaporation: Time-flux-depth patterns. Proc. Soil Sci. Soc. Am. 37: 505-509.

Keulen, H. van, 1975. Simulation of water use and herbage growth in arid regions. Series 'Simulations Monographs'. Pudoc, Wageningen.

Nimah, M. N. \& R. J. Hanks, 1973. Model for estimating soil water, plant and atmospheric interrelations. I. Description and sensitivity. Proc. Soil Sci. Soc. Am. 37: 522-527.

Raats, P. A. C., 1971. Some properties of flows in unsaturated soils with an exponential dependence of hydraulic conductivity upon the pressure head. J. Hydrol. 14: 129-138.

Rijtema, P. E., 1965. An analysis of actual evapotranspiration. Agric. Res. Rep. 659. Pudoc, Wageningen, $107 \mathrm{pp}$.

Staple, W. J., 1971. Boundary conditions and conductivities used in the isothermal model of evaporation from soil. Proc. Soil Sci. Soc. Am. 35: 853-855.

Staple, W. J., 1972. Transport phenomena controlling evaporation from soil. Proc. 2 nd Symp. 'Fundamentals of Transport Phenomena in Porous Media' (7-11 Aug. 1972). University of Guelph, Guelph, Ontario, Canada, p. 566-573.

(continued on p. 82) 


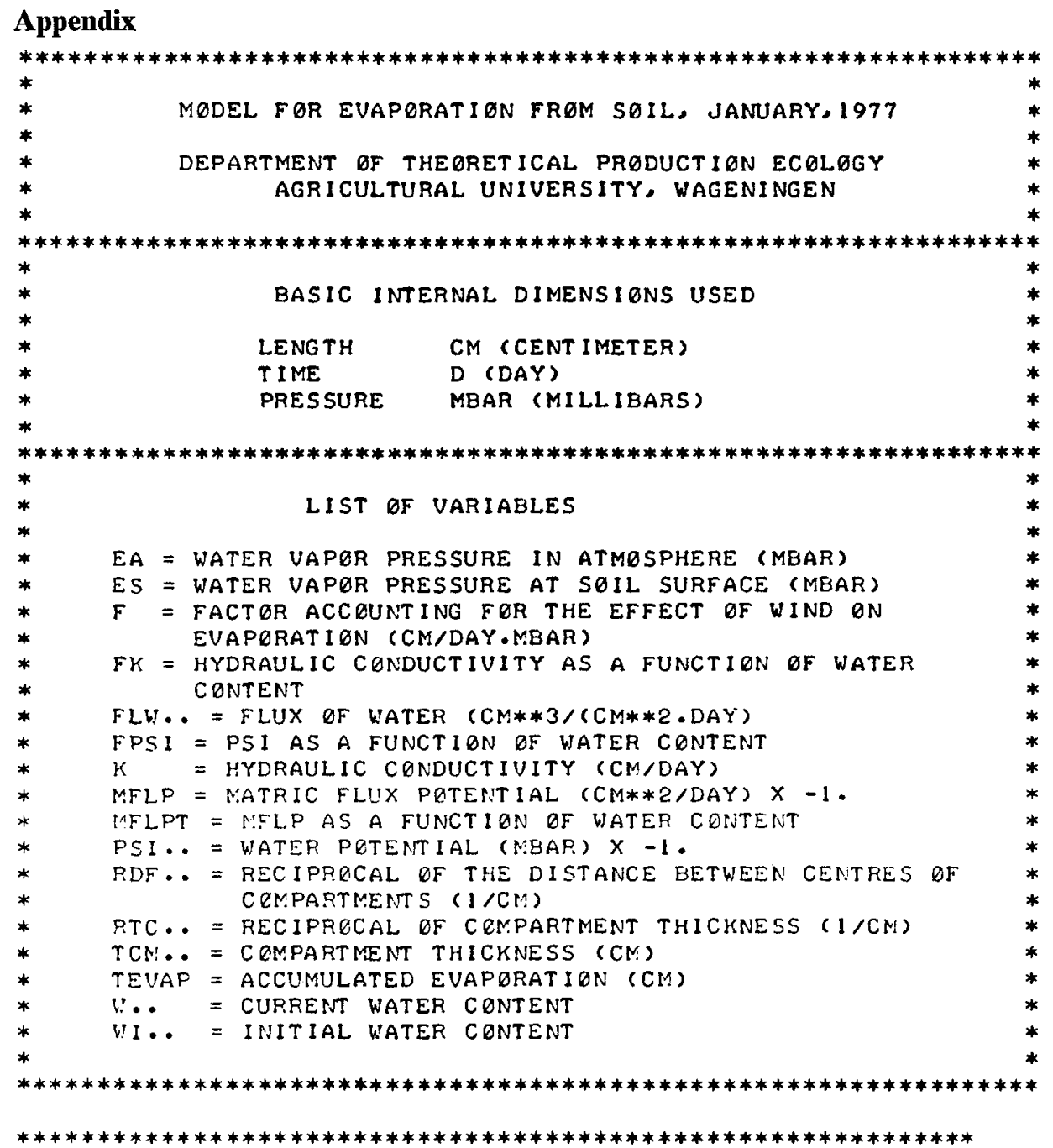

TITLE MODEL FOR EUAPORATION FROM SOIL

TITLE ADELANTO LOAM, JACKSEN (1973)

*****************) DEFINITION OF COMPARTMENT SIZE **************

PARAMETER TCM'1,18* =5*1.,5*1.5,3*2.5,4*5.,1*10. RTC'1,18' = $1, \pi \mathrm{TCM}^{\circ} 1,18^{\circ}$

RDFl $=2 \cdot / \mathrm{TCMI}$

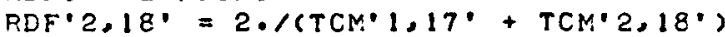

RDF $19=2 \cdot 1 \mathrm{TCM} 18$

******************* INITIAL WATER C INTENT $* * * * * * * * * * * * * * * * * *$

PARAMETER WI'1,18'=18*.2925 


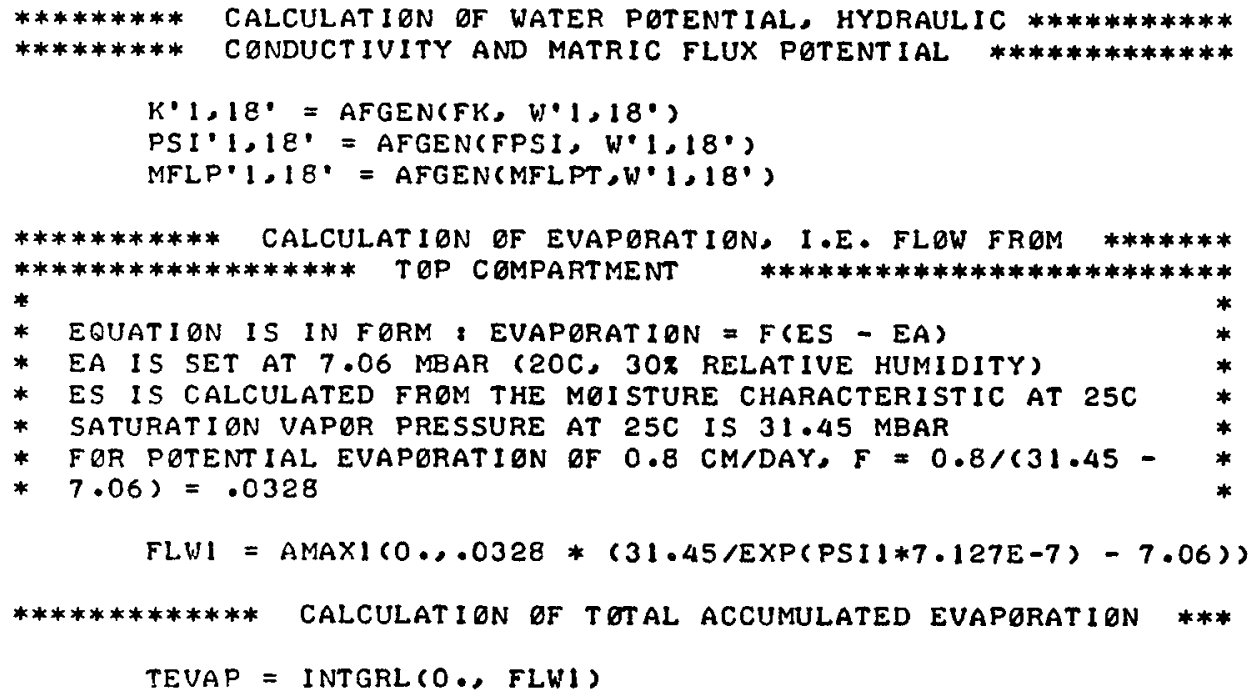


FUNCT I GN TABLES

* matric flux potential (X -1.) as a function of water content

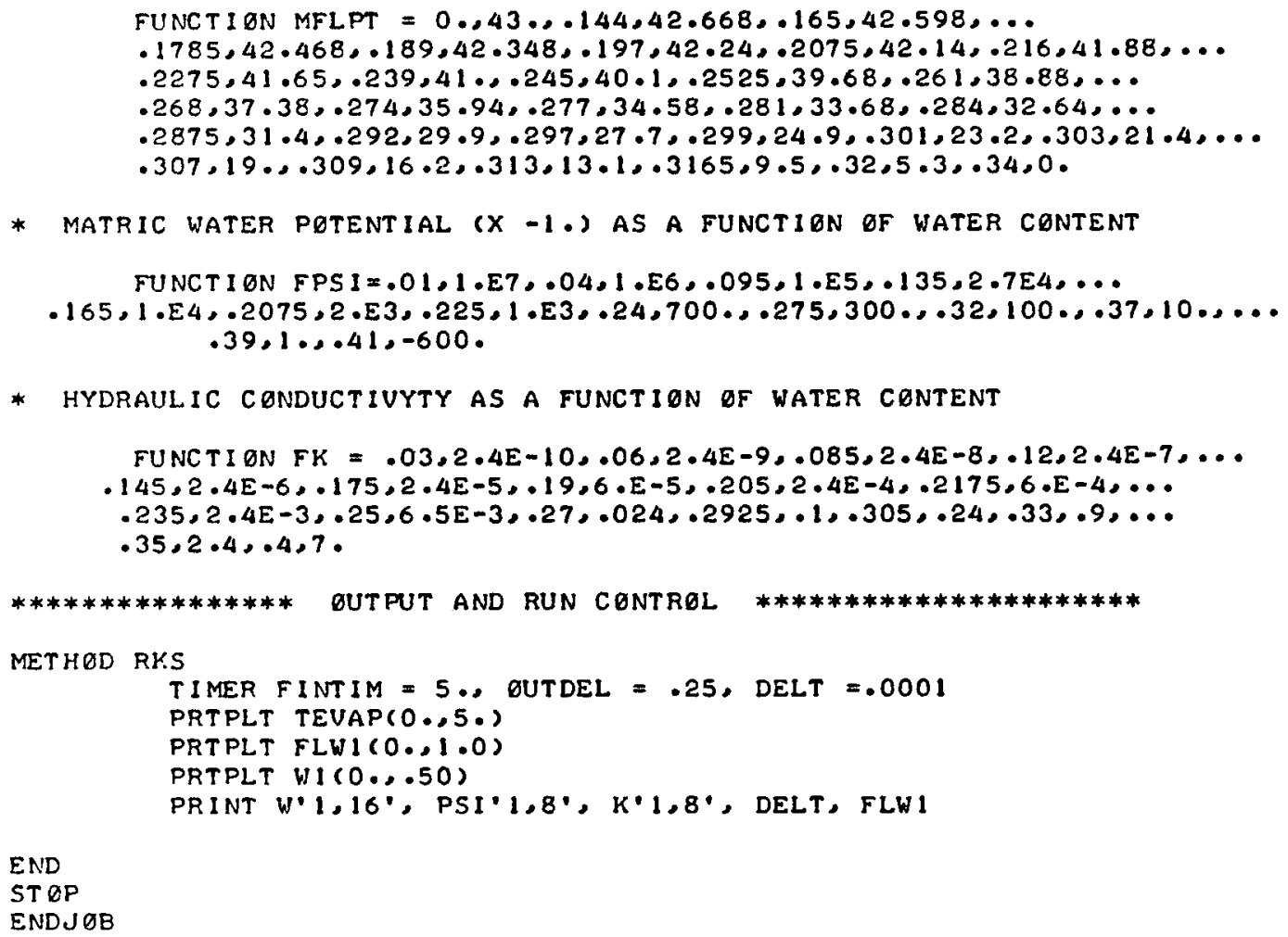

END

ST QP

ENDJ $\boxminus B$

References (continued)

Stroosnijder, L., 1976. Infiltratie en herverdeling van water in grond. Agric. Res. Rep. 847. Pudoc, Wageningen. $213 \mathrm{pp}$.

Warrick, A. W., 1974. Time-dependent linearized infiltration. I. Point sources. Proc. Soil Sci. Soc. Am. 38: 383-386.

Wit, C. T. de \& H. van Keulen, 1972. Simulation of transport processes in soils. Series 'Simulation Monographs'. Pudoc, Wageningen. 